[Agr. Biol. Chem., Vol. 26, No. 11, p. 777 782, 1962]

\title{
Polarographic Studies on Fat-soluble Vitamins in Nonaqueous Media
}

\author{
Part III*. Mechanisms of Electrode-reductions of Vitamin A and its \\ Related Compounds in Acetonitrile-Benzene Mixture as Solvent
}

By Reiji TAKahashi and Isamu TACHI

Department of Agricultural Chemistry, Faculty of Agriculture, Kyoto University, Kyoto

Received August 20, 1962

\begin{abstract}
Polarographic and spectrophotometric examinations on reduction-products obtained by electrolyses at controlled potentials led to the following interpretations in electrode-reductions of vitamin A and its related compounds in acetonitrile-benzene mixture. Each of vitamin Aalcohol and -palmitate is reduced to a structure of conjugated four double-bond system at the first step, and then, at the second step to a conjugated three double-bond system. The first reduction-products of $\beta$-carotene are not uniform and their structures cannot be clarified, but the second reduction-products show absorption spectra of conjugated three double-bond system, consequently, products by the third reduction-step would have a structure of conjugated two double-bond.
\end{abstract}

\section{INTRODUCTION}

In Part $\mathrm{II}^{*}$ of this series of works, a discovery of the polarographic reduction-waves of vitamin $\mathrm{A}$ and its related compounds in acetonitrile-benzene mixture as solvent was described. These compounds have conjugated polyene structures that may be reduced at the dropping mercury electrode $\mathrm{e}^{1 \sim 4)}$. On the other hand, lengths of conjugated polyene systems have been closely related to their absorption spectra in spectrophotometric studies. When a conjugated polyene system is reduced, the length of the system is shortened, which must be shown in a shift of the

\footnotetext{
* Part II.: This Journal, 26, 771 (1962).

1) M. von Stackerberg und W. Strache, Z. Elektrochem., 53, 118 (1949).

2) G.J. Hoijtink and J. van Shooten, Rec. Trav. Chim., 71, 1089 (1952).

3) G.J. Hoijtink and J. van Shooten, ibid., 72. 691 (1953).

4) S. Wawzonek, E.W. Blaha, R. Berkey and M.E. Runner, J. Electrochem. Soc., 102, 235 (1955).
}

absorption-band of the resultant reductionproduct to the shorter wave-length. Thus, the present purpose is to study the reductionmechanisms of vitamin $\mathrm{A}$ and its related compounds by means of polarography and spectrophotometry on the reduction-products generated by electrolyses at the constant potentials where the polarographic reductionwaves occur under the conditions described previously.

\section{EXPERIMENTAL}

Materials and chemicals, vitamin A-alcohol, -palmitate, $\beta$-carotene, tetrabutylammonium iodide, acetonitrile and benzene, were prepared in the same way as the previous study.

The basal constitution of electrolytic solutions for polarographic measurements, 60 vol\% benzene-acetonitrile mixture containing $0.1 \mathrm{M}$-tetrabutylammonium iodicle, was the same as the previous, and it was also the same as basal constitution of electrolytic solutions 
for electrolyses at controlled potentials so that the potential data obtained in the previous study were applicable in the present electrolyses executed with the mercury electrodes in those electrolytic solutions. All the experimental conditions were also the same as the previous; in particular, it is important to protect those experiments against actions of atmospheric oxygen and light*.

Electrolyses at controlled potentials were carried out with a vessel, net volume of ca. $5 \mathrm{ml}$, which had two mercury-pools for cathode and reference electrode, respectively. Anode, which consisted of a large mercury-pool in the same electrolytic solution, was set outside the vessel and connected through a liquid junction to make generated reduction-products free from contaminations by oxidation-products. The mercury cathode and the electrolytic solution were stirred by nitrogen-bubbling during electrolysis. An electrolysis was regarded as being brought to completion when electrolytic current reached to a small and constant value; the time required was commonly about two or three hours at a given concentration of depolarizer, which stood between $10^{-4} \mathrm{M}$ and $10^{-3} \mathrm{M}$.

When a polarogram of electrolysis-product was required, the solution in electrolysis-vessel was directly electrolyzed with dropping mercury electrode against mercury-pool electrode.

In determination of absorption spectra, above electrolytic solution was diluted to ca. $10^{-5} \mathrm{M}$, as for the subject with pure acetonitrile, and used. The blank solution was prepared in the same way with blank solution for electrolysis. In the range of adopted wave-length, from $270 \mathrm{~m}_{\mu}$ to $550 \mathrm{~m} \mu$, any absorption spectra of both acetonitrile and supporting electrolyte were not observed; absorption of coexistent benzene occurred at shorter wave-length than $270 \mathrm{~m}_{\mu}$.

Electrolyses at controlled potentials were carried out with modified polarographic cell and the Yanagimoto Automatic Controlled Potential Electrolyser VE3. Polarograms were obtained with the Yanagimoto Polarograph PA-2. Determinations of absorption spectra were executed with conventional quartz cell and the Hitachi Pen-recording Spectrophotometer EPS-2.

\footnotetext{
* Especially vitamin A-alcohol and $\beta$-carotene are quite sensitive to both oxygen and light, and simply degenerate into inactive and somewhat less soluble oxidation-products.
}

\section{RESULTS AND DISCUSSION}

(1) Resultant Polarograms from Electrolyses at Constant Potentials and Corresponding Absorption Spectra.

Vitamin A-alcohol. The first electrolysis of vitamin A-alcohol was carried out at constant potential of $-1.45 \mathrm{~V}$ vs. $\mathrm{Hg}$ (reference electrode of mercury in the electrolysis vessel; where the polarographic reduction-current of the first wave almost reached to its limiting value and that of the second wave did not yet occur, and the resultant reduction-product showed a polarographic reduction-wave which lacked only the first wave as shown in Fig. 1 (left). The second electrolysis was carried out at constant potential of $-1.90 \mathrm{~V}$, and the resultant reduction-product showed a polarographic reduction-wave which consisted of only the third wave, being made more distinct by these electrolyses. Result obtained by direct electrolysis at $-1.90 \mathrm{~V}$ was the same as the result from above consecutive electrolyses.

Absorption spectra of these reduction-products are illustrated in Fig. 2 (left). Wavelength of the maximum absorption of vitamin A-alcohol in the given medium, $32_{8} \mathrm{~m} \mu$, was slightly shifted to short value, $32_{1} \mathrm{~m} \mu$, by the first electrolysis; at the same time the extinction decreased. After the second electrolysis, the absorption-band shifted to shorter value in wave-length and two shoulders appeared at $29_{2} \mathrm{~m} \mu$ and $28_{1} \mathrm{~m} \mu$. Measurement in shorter wave-length than $270 \mathrm{~m} \mu$ was impossible because of coexistence of small amount of benzene.

Vitamin A-palmitate. Each of the fixed potentials at which vitamin A-palmitate was electrolyzed stepwise was the same as each potential in the case of vitamin A-alcohol. As illustrated in Figs. 1 (right) and 2 (right), polarograms and absorption spectra of the resultant reduction-products showed almost the same characteristics as those of vitamin A-alcohol. 
Polarographic Studies on Fat-soluble Vitamins in Nonaqueous Media. Part III

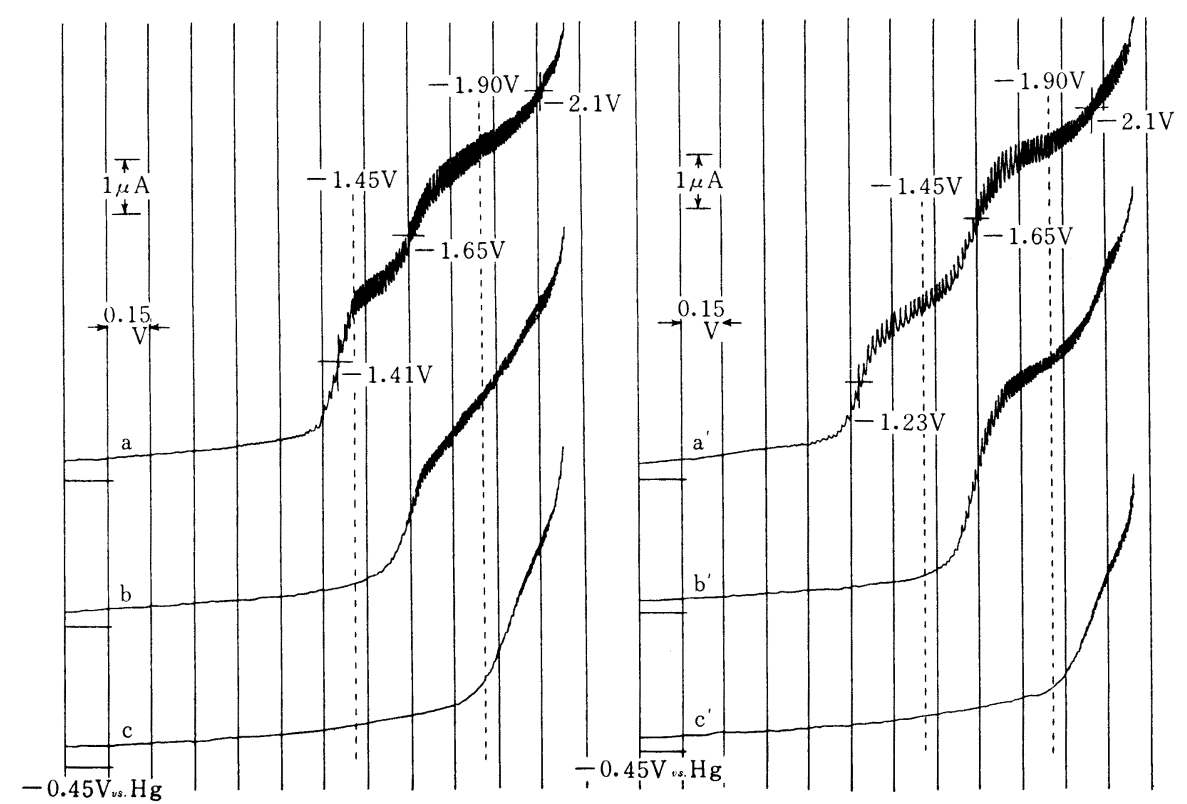

FIG. 1. D. G. Polarograms of Vitamin A-alcohol (left, $5 \times 10^{-4} \mathrm{M}$ ) and Vitamin A-palmitate (right, $7.5 \times 10^{-4} \mathrm{M}$ ) in $60 \mathrm{vol} \%$ Benzene-Acetonitrile Containing $0.1 \mathrm{M}-\mathrm{Bu}_{4} \mathrm{NI}$.

(a, $\mathrm{a}^{\prime}$ ) before Electrolyses, $\left(\mathrm{b}, \mathrm{b}^{\prime}\right.$ ) after Electrolyses at Constant Potential of $-1.45 \mathrm{~V}$ vs. $\mathrm{Hg},\left(\mathrm{c}, \mathrm{c}^{\prime}\right)$ after Electrolyses at Constant Potential of $-1.90 \mathrm{~V}$ vs. Hg.
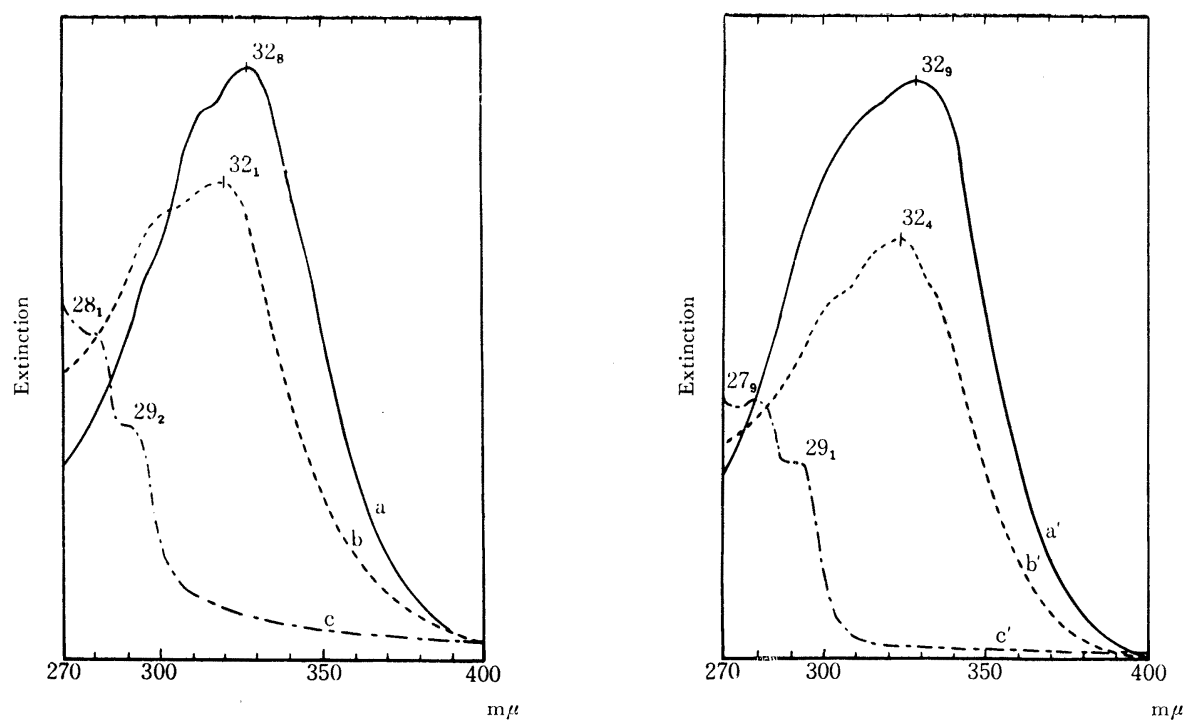

FIG. 2. Absorption Spectra of Vitamin A-alcohol (left, $2.5 \times 10^{-5} \mathrm{M}$ ) and Vitamin A-palmitate (right, $2.5 \times 10^{-5} \mathrm{M}$ ) in Acetonitrile.

(a, a') before Electrolyses, (b, b') after Electrolyses at Constant Potential of $-1.45 \mathrm{~V} \mathrm{vs.} \mathrm{Hg},\left(\mathrm{c}, \mathrm{c}^{\prime}\right.$ ) after Electrolyses at Constant Potential of $-1.90 \mathrm{~V}$ vs. $\mathrm{Hg}$. 
$\beta$-Carotene. In the case of $\beta$-carotene, the fixed potential of the first electrolysis was $-1.25 \mathrm{~V}$ and that of the second was $-1.80 \mathrm{~V}$. Resultant polarogram from the first electrolysis, being illustrated in Fig. 3 (left), showed a series of small reduction-waves, and the corresponding absorption spectrum illustrated in Fig. 4 also showed a series of small absorption maxima in the range from $340 \mathrm{~m} \mu$ to $400 \mathrm{~m} \mu$, though the absorption-band shifted

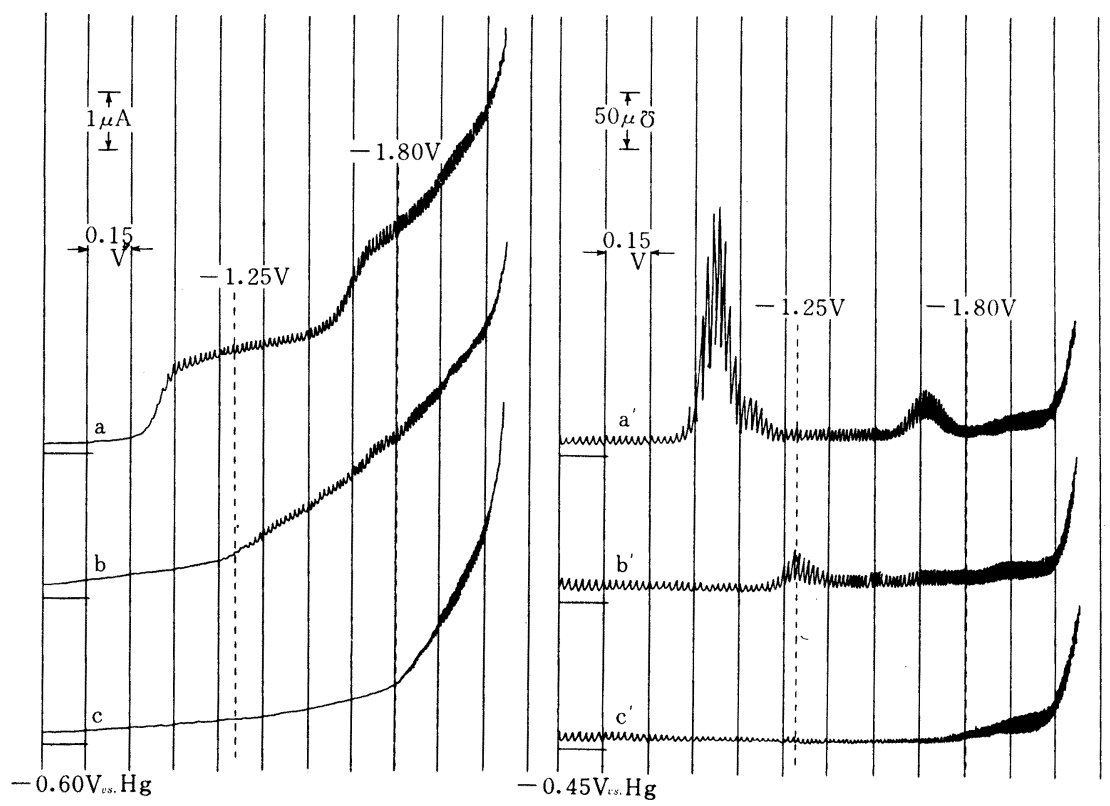

FIG. 3. D.G. (left) and A.G. (right) Polarograms of $\beta$-Carotene $\left(2.5 \times 10^{-4} \mathrm{M}\right)$ in $60 \mathrm{vol} \%$ Benzene-Acetonitrile Containing $0.1 \mathrm{M}-\mathrm{Bu}_{4} \mathrm{NI}$.

$\left(\mathrm{a}, \mathrm{a}^{\prime}\right)$ before Electrolysis, $\left(\mathrm{b}, \mathrm{b}^{\prime}\right)$ after Electrolysis at Constant Potential of $-1.25 \mathrm{~V}$ vs. $\mathrm{Hg},\left(\mathrm{c}, \mathrm{c}^{\prime}\right)$ after Llectrolysis at Constant Potential of $-1.80 \mathrm{~V}$ vs. $\mathrm{Hg}$.

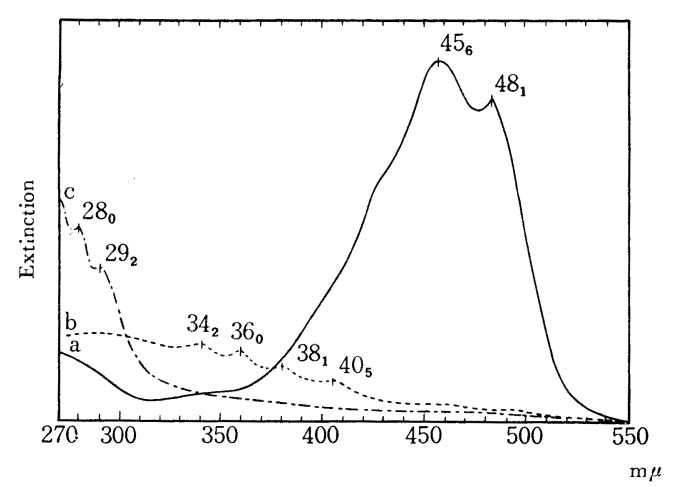

FIG. 4. Absorption Spectra of $\beta$-Carotene $\left(5 \times 10^{-6} \mathrm{M}\right)$ in Acetonitrile.

(a) before Electrolysis, (b) after Electrolysis at Constant Potential of $-1.25 \mathrm{~V}$ vs. $\mathrm{Hg}$, (c) after Electrolysis at Constant Potential of $-1.80 \mathrm{~V}$ vs. $\mathrm{Hg}$. to shorter value in wave-length and the extinction decreased extremely. As also illustrated in Figs. 3 and 4, resultant polarogram from the second electrolysis showed only the third wave of the original reduction-polarogram and the corresponding absorption spectrum were almost the same as corresponding spectra of vitamin A-alcohol and -palmitate. The direct electrolysis at the fixed potential of the second electrolysis described above gave the same results. In Fig. 3 (right), the corresponding a.c. polarograms are illustrated.

(2) Half-empirical Treatments on Absorption Maxima.

In the calculations of the wave-lengths, 
$\lambda_{\max }$, at which absorption maxima of the main absorption-bands occur, the next half-empirical equation ${ }^{5)}$ was employed.

$$
\begin{aligned}
\lambda_{\max } & =\sqrt{A-B \times 0.920^{N}} \times 10^{2} \mathrm{~m} \mu \\
N & =N_{c}+\sum N_{a}
\end{aligned}
$$

where $A$ and $B$ are constants based on adopted solvent, $N_{c}$ is the number of conjugated double-bonds and $N_{a}$ is the effect of auxochromous groups involved in polyene molecule. Related $N_{a}$-values are listed in Table I. Both $A$ and $B$ in the given solvent can be evaluated with the wave-lengths at absorption maxima of two compounds whose $N$-values are known. It was done with vitamin A-palmitate $\left(\lambda_{\max }=32_{9} \mathrm{~m} \mu, N=4.8\right)$ and $\beta$-carotene $\left(\lambda_{\max }=45_{6} \mathrm{~m} \mu, N=10.4\right)$, and the resultant expression was as follows.

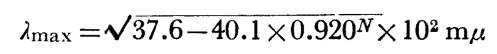

The results of calculations with this equation are introduced and discussed in the next paragraph.

TABle I. EFFeCt OF AuXochromous GRouP

\begin{tabular}{|c|c|}
\hline Radical or Structure & Effect $(\mathrm{N} a)$ \\
\hline Alkyl & +0.1 \\
$\alpha$-Oxyalkyl & +0.1 \\
Alkoxyl & 0 \\
& +0.1 \\
\hline
\end{tabular}

(3) Reduction Mechanisms.

It was described in the last paper of this series of works that each of vitamin A-alcohol and -palmitate accepts two electrons at the first reduction-step and two more electrons at the second, while $\beta$-carotene accepts four electrons at the first and four more at

5) K. Hirayama, J. Chem. Soc. Japan, 75, 29, 667, 674 (1954). the second. Those data and the present polarographic and spectrophotometric considerations led to the following interpretations on these reduction mechanisms.

Vitamin A-alcohol. The fact that shift of absorption maximum is small denies that the conjugated double-bond system is divided into two independent systems by the first twoelectron reduction. Thus, the possible structures of the first reduction-product, which must be anion free-radical at first and then hydrogenated under the conditoins, are the three shown below (I, II and III).

I.

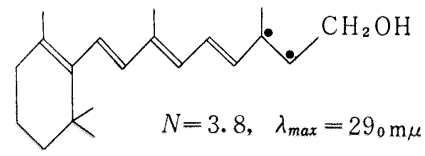

II.

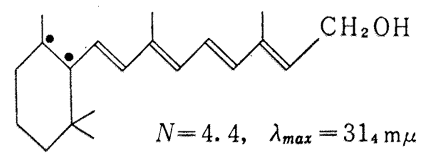

III.

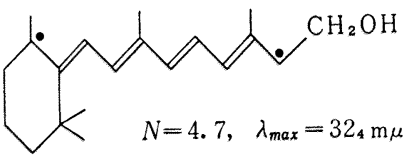

where $\lambda_{\max }$-values were calculated with Eqn. (2). Observed $\lambda_{\max }$ was $32_{1} \mathrm{~m} \mu$, so that it corresponds to that of the structure III. Two absorbed electrons which are negative may be situated as apart as possible. They are possibly absorbed at each of two ends of conjugated system, respectively, because the conjugated system of vitamin A-alcohol is not so long.

As treated above, $\lambda_{\max }$-values of possible structures of conjugated three double-bond system which may be generated by the second two-electron reduction were calculated, and the resutlant were in the range from $240 \mathrm{~m} \mu$ to $280 \mathrm{~m} \mu$. The two observed peaks or shoulders, $28_{1} \mathrm{~m} \mu$ and $29_{2} \mathrm{~m} \mu$, are approxi- 
mately corresponding with the calculated.

Consequently, vitamin A-alcohol accepting two electrons acquires the above described structure of conjugated four double-bond at the first reduction-step, and then, at the second reduction-step it accepts two more electrons and is reduced to a conjugated three double-bond system which will be reduced further at the third reduction-step to a conjugated two double-bond system.

Vitamin A-palmitate. The palmityl radical esterified with vitamin A-alcohol scarcely influenced the absorption-band of vitamin Aalcohol, and the calculated $\lambda_{\max }$-values were all the same as the correspondings of vitamin A-alcohol. On the other hand, saturated esters were not reduced at dropping mercury electrode in the present medium. Therefore the reduction mechanism of vitamin A-palmitate must be the same as that of vitamin Aalcohol. Polarograms and absorption spectra of the reduction-products support the above interpretation.

$\beta$-Carotene. Both polarogram and absorption spectrum of the products generated by the first four-electron reduction of $\beta$-carotene were very complicated. It shows the first reduction-products are not uniform. In this case it is impossible to calculate the absorption maxima with such simple method as employed above.

The second reduction-products absorbed four more electrons showed only the third reduction-wave in polarogram, and their absorption spectra were quite similar to the correspondings of vitamin A-alcohol and -palmitate. This shows that almost all the second reduction-products have conjugated three double-bond systems, each of which will also cause the third reduction-step in the same way as in the cases of vitamin A-alcohol and -palmitate. 